\title{
Marcadores de inflamación endotelial subclínica en una familia con hiperaldosteronismo familiar tipo I por mutación de novo
}

\author{
Carlos B Stehr ${ }^{1 *}$, Cristián A Carvajal ${ }^{1}$, Patricia Lacourt ${ }^{2}$, \\ Hernán Alcaíno ${ }^{3}$, Rosemarie Mellado ${ }^{3}$, Andreína Cattani2, \\ Lorena M Mosso ${ }^{1}$, Sergio Lavandero ${ }^{3}$, Carlos E Fardella ${ }^{1}$. \\ Subclinical endothelial inflammation \\ markers in a family with type I \\ familial hyperaldosteronism caused \\ by a de novo mutation
}

Background: Type I familial hyperaldosteronism is caused by the presence of a chimaeric gene CYP11B1/CYP11B2 which encodes an enzyme with aldosterone synthetase activity regulated by adrenocorticotrophic hormone (ACTH). Therefore, in patients with FHI is possible to normalize the aldosterone levels with glucocorticoid treatment. Recently it has been shown that aldosterone plays a role in the production of endothelial oxidative stress and subclinical inflammation. Aim: To evaluate subclinical endothelial inflammation markers, like Metalloproteinase 9 (MMP-9) and ultrasensitive $\mathrm{C}$ reactive protein (usPCR), before and after glucocorticoid treatment in family members with FH-I caused by a de novo mutation. Patients and methods: We report three subjects with FH-I in a single family (proband, father and sister). We confirmed the presence of a chimaeric CYP11B1/CYP11B2 gene by long-PCR in all of them. Paternal grandparents were unaffected by the mutation. The proband was a 13 year-old boy with hypertension stage 2 (in agree to The Joint National Committee VII, JNC-VII), with an aldosterone/plasma rennin activity ratio equal to 161. A DNA paternity test confirmed the parental relationship between the grandparents and father with the index case. MMP-9 and usPCR levels were determined by gelatin zymography and nephelometry, respectively. Results: All affected subjects had approximately a 50\% increase in MMP-9 levels. Only the father had an elevated usPCR. The endothelial inflammation markers returned to normal range after glucocorticoid treatment. Conclusions: We report a family carrying a FH-I caused by a de novo mutation. The elevation of endothelial inflammation markers in these patients and its normalization after glucocorticoid treatment provides new insight about the possible deleterious effect of aldosterone on the endothelium (Rev Méd Chile 2008; 136: 1134-40).

(Key words: C-reactive protein; Endothelium, vascular; Hyperaldosteronism; MMP-9 metalloproteinase)

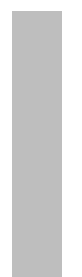

\footnotetext{
Recibido el 27 de noviembre, 2007. Aceptado el 29 de abril, 2008.

Financiamiento: Fondecyt № 1070876. Proyecto de becado UC PG-11/07.

${ }^{1}$ Departamento de Endocrinología, ${ }^{2}$ Departamento de Pediatría, Unidad de Endocrinología Infantil, Facultad de Medicina, Pontificia Universidad Católica de Chile. ${ }^{3}$ Centro FONDAP Estudios Moleculares de la Célula, Facultad de Ciencias Químicas y Farmacéuticas, Universidad de Chile. Santiago, Chile.

*Dr. Carlos Stehr se encuentra realizando residencia en Endocrinología con financiamiento de la Sociedad Chilena de Endocrinología y Diabetes.
}

Correspondencia a: Dr. Carlos E. Fardella. Departamento de Endocrinología, Escuela de Medicina, Pontificia Universidad Católica de Chile. Lira 85, 5o piso, Santiago-Chile. Teléfono: 05602-354 3095. Fax: 056-02-638 5675. E mail: cfardella@med.puc.cl 
$\mathrm{E}^{1}$ hiperaldosteronismo primario (HAP) se debe a la producción autónoma de aldosterona por la zona glomerulosa de la glándula suprarrenal, siendo su secreción independiente del eje renina angiotensina. Es una de las causas más frecuentes de hipertensión arterial (HTA) secundaria, habiéndose informado una incidencia de $5 \%$ a $20 \%$ de HAP en la población de hipertensos, al utilizar la relación de aldosterona plasmática/actividad de renina plasmática como método de tamisaje ${ }^{1}$. La aldosterona, además de producir daño endotelial a través de la hipertensión arterial, produce efectos directos sobre varios órganos, describiéndose daño a nivel del corazón y endotelio, lo que se traduce en fibrosis miocárdica y disfunción endotelial. Se ha demostrado que los pacientes con HAP tienen más hipertrofia ventricular izquierda y mayor cantidad de eventos cardiovasculares que los esperados para su nivel de presión arterial al compararlos con hipertensos esenciales ${ }^{2}$.

Existen diversos marcadores de inflamación endotelial subclínica, entre los cuales, la proteína $C$ reactiva ultrasensible (PCRus) ha sido uno de los más utilizados y validados como marcador de riesgo cardiovascular en pacientes con cardiopatía coronaria $^{3}$. Estudios recientes han demostrado que en pacientes con angina estable, niveles elevados de PCRus predicen en forma independiente un mayor riesgo de eventos cardiovasculares $^{4}$. El aumento de PCRus también ha sido estudiado en otras poblaciones de pacientes, entre los cuales se encuentran aquellos portadores de síndrome metabólico ${ }^{5,6}$.

Las metaloproteinasas corresponden a una familia de endoproteinasas que degradan componentes de la matriz extracelular, siendo su transcripción estimulada por numerosas citoquinas inflamatorias, como interleuquinas 1 y 6 , factor de crecimiento derivado de plaquetas (PDGF), CD40 y factor de necrosis tumoral (TNF) ${ }^{7,8}$. Se ha demostrado un aumento de los niveles de metaloproteinasa 9 (MMP-9) en sujetos con angina estable al compararlos con controles sanos ${ }^{9}$, observándose además en un estudio prospectivo que pacientes con cardiopatía coronaria que presentaron un evento cardiovascular fatal tenían niveles más elevados de MMP-910.

La xantina oxidasa cataliza el paso de hipoxantina a xantina. Su actividad está elevada en procesos en los cuales hay un aumento del estrés oxidativo endotelial. Una mayor actividad de esta enzima incrementa la producción de ión superóxido y de especies reactivas derivadas del oxígeno (ROS), con la consiguiente oxidación de moléculas a nivel celular ${ }^{11}$. Una de las estructuras celulares que experimenta oxidación por las ROS es la membrana celular, generándose productos de lipoperoxidación, algunos de los cuales pueden ser medidos ${ }^{12,13}$. El estrés oxidativo además activa distintas vías de transducción de señales intracelulares, activando factores de transcripción como son el factor nuclear NF-kB, el activador de proteína (AP)-1 y CREB. La unión de estos factores de transcripción a los promotores de sus genes blanco aumenta la transcripción de diversos genes, entre ellos los que codifican para citoquinas proinflamatorias $^{14}$. Estudios experimentales han mostrado que la aldosterona induciría estrés oxidativo a través de la activación del receptor de mineralocorticoides, estimulando la generación de ROS y moléculas proinflamatorias ${ }^{15-17}$.

El HAF-I se produce por una recombinación desigual entre los genes que codifican para la $11 ß$ hidroxilasa (CYP11B1) y la aldosterona sintetasa (CYP11B2), resultando en un gen quimérico cuyo producto tiene actividad de aldosterona sintetasa, pero que es regulado por ACTH ${ }^{18-20}$. Dado que la producción de aldosterona en el HAF-I se encuentra bajo el control de la ACTH es posible tratarlo administrando glucocorticoides y de esta manera normalizar los niveles de aldosterona plasmática, constituyendo por lo tanto un modelo ideal para evaluar los efectos directos de aldosterona sobre la disfunción endotelial.

Recientemente, nuestro grupo de investigación detectó una familia en la cual 3 sujetos son portadores de hiperaldosteronismo familiar tipo I por una mutación de novo, observando en ellos un aumento del estrés oxidativo endotelial, el cual regresaba con el tratamiento específico de esta patología ${ }^{21}$. El objetivo del presente estudio es evaluar marcadores de inflamación endotelial subclínica previo y post tratamiento específico con glucocorticoides en 3 sujetos portadores de HAF-I por una mutación de novo.

\section{PACIENTES Y MÉTODOS}

Pacientes. Las características clínicas de los pacientes estudiados se describieron ampliamente en un estu- 
dio publicado recientemente por nuestro grupo ${ }^{21}$. El caso índice corresponde a un varón de 13 años, el cual es parte de una familia conformada por 29 integrantes consanguíneos por línea paterna (12 tíos, 11 primos, padre, 2 hermanas y 2 abuelos), todos ellos vivos. Consultó por HTA (180/110 mmHg), encontrándose niveles plasmáticos de aldosterona (AP) de 48,4 ng/dL, actividad de renina plasmática (ARP): $\varangle 0,2 \mathrm{ng} / \mathrm{mL} \mathrm{h}$ y relación AP/ARP: 161,3 , compatible con HAP. A su padre se le diagnosticó HAP a los 25 años de edad en relación a HTA etapa 2 (JNC 7) e hipokalemia severa, actualmente en tratamiento con amilonide, AP: 69,4 ng/dL, ARP: 1,5 ng/mL h, relación AP/ARP: 46,2, potasio plasmático normal. Tiene 2 hermanas de 16 y 15 años, ambas normotensas y sin antecedentes mórbidos de importancia. La hermana de 15 años, AP: 11,3 ng/dL, ARP: $\varangle 0,2 \mathrm{ng} / \mathrm{mL}$ h, relación AP/ARP: 37,6.

Para confirmar la existencia de un HAF-I se realizó estudio genético para gen quimérico CYP11B1/CYP11B2 al caso índice y familiares directos (padre, hermanos, abuelos paternos y 6 tíos paternos), encontrándose afectados el caso índice, padre y hermana de 15 años. No se pesquisó el gen quimérico en otros integrantes de la familia, siendo ambos abuelos paternos negativos para la mutación.

En los 3 casos en los que se confirmó el diagnóstico de HAF-I, antes y 2 meses tras el inicio del tratamiento con glucocorticoides se evaluaron presión arterial, AP, ARP, potasio plasmático, MMP-9 y PCRus. Se inició tratamiento específico con dexametasona en el padre $(0,5 \mathrm{mg} /$ día vo) y hermana $(0,25 \mathrm{mg} /$ día vo). En el caso índice, por encontrarse aún en periodo de crecimiento, se indicó tratamiento con cortisol $(10 \mathrm{mg} /$ $\mathrm{m}^{2}$ superficie corporal fraccionado en 3 dosis diarias) para evitar una supresión prolongada del eje suprarrenal y de este modo no interferir con la talla final. Se obtuvo consentimiento informado de todos los participantes de acuerdo a las guías de la Declaración de Helsinki y la aprobación del comité de ética de la Facultad de Medicina de la Pontificia Universidad Católica de Chile.

Evaluación bioquímica. La AP se midió por radioinmunoensayo directo utilizando un kit comercial (Diagnostic Products, Los Angeles, CA). La variación intra e interensayo fue 5,1\% y 7,1\%, respectivamente, siendo el valor normal entre $1-16 \mathrm{ng} / \mathrm{dL}$.
La ARP se evaluó por radioinmunoanálisis de angiotensina I obtenida por generación enzimática, usando el kit comercial DIASORIN con una variabilidad intra e interensayo de 6,1\% y 6,6\%, respectivamente. Su rango normal se encuentra entre 1,3-4 $\mathrm{ng} / \mathrm{mL}$ h. El límite inferior de determinación de ARP fue de $0,2 \mathrm{ng} / \mathrm{mL} \mathrm{h}$. El potasio plasmático se midió por potenciometría indirecta ión selectivo en equipo automatizado HITACHI, siendo el rango de referencia de 3,5-5,0 $\mathrm{mEq} / \mathrm{L}$.

Evaluación de inflamación endotelial subclínica. La determinación de actividad de la MMP-9 se realizó mediante zimografía en geles de 10\% poliacrilamida-SDS copolimerizado con $1 \mathrm{mg} / \mathrm{mL}$ de gelatina de acuerdo a lo descrito por Kameda et $\mathrm{al}^{22}$. Las actividades se evaluaron por densitometría y se expresaron respecto a una actividad de MMP-2 estándar (Sigma). Las variaciones inter e intraensayo fueron de $5 \%$ y $8 \%$, respectivamente; siendo el rango de referencia entre 0,01 a 0,22 veces MMP-2 estándar. Los valores de referencia de MMP-9 se calcularon a partir de 12 controles sanos (10 hombres y 2 mujeres) con una edad promedio de $60 \pm 12$ años, los cuales no tenían antecedente de hipertensión arterial, enfermedad cardiovascular, tabaquismo $\mathrm{u}$ otro factor de riesgo cardiovascular que pudiera alterar los resultados de estos marcadores. La PCRus se determinó por nefelometría con un coeficiente de variación inter e intraensayo de 3,5\%. El valor de referencia normal es menor a $3 \mathrm{mg} / \mathrm{L}$.

Estudio genético. Este se realizó siguiendo el protocolo descrito por Jonsson ${ }^{23}$. La presencia del gen quimérico CYP11B1/CYP11B2 y del gen de la aldosterona sintetasa CYP11B2 se determinó extrayendo el ADN genómico de leucocitos de sangre periférica. El ADN aislado de cada paciente se sometió a 2 reacciones de amplificación realizadas simultáneamente mediante XL-PCR. En la primera reacción el primer sense ( 5 'TCC TTC ATC TAC CTT TGG CTG GGG 3') es específico para la región 5' del gen de la aldosterona sintetasa. En la segunda reacción el primer sense ( 5 'TCA TGC ACC CCC AAT CAG TCC CTG $3{ }^{\prime}$ ) es específico para la región 5 ' del gen de 11ß-hidroxilasa. En ambas reacciones el primer antisense ( 5 'GAG TCC TCC AGC TGC CTC TCA ACC $3^{\prime}$ ) es específico para la región del intrón $\mathrm{E}$ del gen de la aldosterona sintetasa. Una 
nueva reacción de amplificación en el caso índice, su padre, hermana y abuelos paternos se realizó siguiendo la misma metodología descrita anteriormente pero utilizando primers dirigidos contra otras zonas del gen quimérico CYP11B1/CYP11B2 y gen de la aldosterona sintetasa ${ }^{24}$. Los primers están dirigidos contra regiones de la CYP11B1 y CYP11B2 que no son homólogas entre ellas, permitiendo la especificidad de la reacción. En cada reacción se incluyó un control positivo y negativo para confirmar la especificidad de la reacción. El producto de amplificación se visualizó en transiluminador-UV tras electroforesis en gel de agarosa 0,8\% con bromuro de etidio.

Estudio de paternidad. Las muestras de ADN genómico del padre y abuelos del caso índice se analizaron utilizando el ensayo comercial Identifiler (Applied Biosystems), que incluye el análisis de 15 marcadores genéticos (unidades cortas repetidas en tándem o STR) y el gen de la amelogenina para identificar el sexo en un equipo de electroforesis capilar ABI 310 (Applied Biosystems).

\section{RESULTADOs}

Estudio genético. Sólo el padre, hermana y caso índice demostraron ser positivos para la mutación, no pesquisándose la mutación en los tíos ni abuelos paternos estudiados (Figura 1). La ausencia de amplificación del gen quimérico en ambos abuelos paternos tanto en la primera como segunda reacción de XL-PCR descarta la presencia de un polimorfismo en el sitio de unión de los primers al segmento específico del gen en estudio.

Estudio de paternidad. Debido a que sólo el caso índice, su padre y hermana eran portadores del gen quimérico CYP11B1/CYP11B2, siendo sus abuelos paternos negativos para la mutación, se realizó estudio de paternidad con el objetivo de aclarar la relación parental entre abuelos y padre del caso índice. El estudio de paternidad mostró una correlación perfecta entre las secuencias hipervariables analizadas en cada uno de ellos certificando la relación parental entre ellos.

Marcadores de inflamación endotelial subclínica. En relación a los marcadores de inflamación endotelial subclínica se observó una elevación de la PCRus sólo en el padre $(4,08 \mathrm{mg} / \mathrm{L})$, mientras que los niveles de MMP-9 se encontraron en todos ellos elevados al doble del límite superior del rango de referencia (Tabla 1 ).

Tras 2 meses de tratamiento con glucocorticodes se observó una normalización de los niveles de aldosterona plasmática en los 3 pacientes y una relación AP/ARP normal en el padre y hermana. En el caso índice la relación se mantuvo levemente elevada en relación a una ARP suprimida. Se

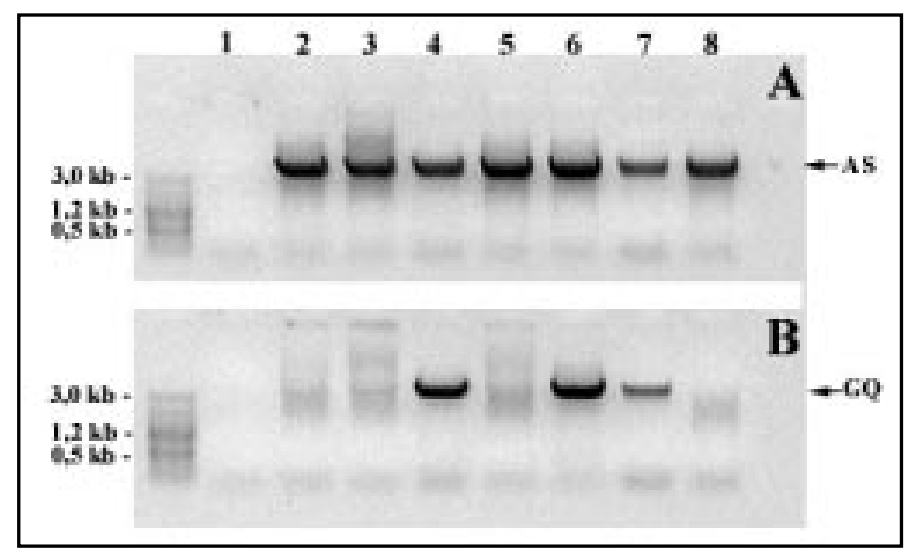

Figura 1. Amplificación por XL-PCR de gen quimérico CYP11B1/CYP11B2 (GQ) y gen de la aldosterona sintetasa CYP11B2 (AS) en ADN de leucocitos de caso índice, padre, 2 hermanas y abuelos paternos. Columnas de derecha a izquierda: $1 . \mathrm{H}_{2} 0$, 2. Abuelo paterno, 3. Abuela paterna, 4. Padre, 5. Hermana sana, 6. Hermana portadora, 7. Caso índice, 8. Control negativo. 


\section{Tabla 1. M arcadores de inflamación endotelial subclínica pre y post tratamiento en pacientes con hiperaldosteronismo familiar tipo I}

\begin{tabular}{|c|c|c|c|c|c|c|c|}
\hline & & $\begin{array}{l}\text { P Art } \\
\mathrm{mmHg}\end{array}$ & $\begin{array}{l}\text { AP/ARP } \\
\text { VR: }<25\end{array}$ & $\begin{array}{c}\text { PCRus } \\
\text { VR: }<3 \mathrm{mg} / \mathrm{L}\end{array}$ & $\begin{array}{c}\text { MMP-9 } \\
\text { VR:0,01-0,22 } \\
\text { veces MMP9 std }\end{array}$ & $\begin{array}{c}\text { XO } \\
\text { VR:0,06-0,23 } \\
\text { UAxmL }^{-1} \text { X min }^{-1}\end{array}$ & $\begin{array}{c}\text { MDA } \\
\text { VR:0,3-1,1 } \mu \mathrm{M}\end{array}$ \\
\hline \multirow[t]{2}{*}{ Caso índice } & Pre & $110 / 60$ & 161 & 0,15 & 0,39 & 0,23 & 2,3 \\
\hline & Post & $130 / 80$ & 29 & 0,41 & 0,20 & 0,14 & 1,1 \\
\hline \multirow[t]{2}{*}{ Padre } & Pre & $130 / 80$ & 46,2 & 4,08 & 0,35 & 0,25 & 2,1 \\
\hline & Post & $129 / 83$ & 1,64 & 0,84 & 0,12 & 0,15 & 1,2 \\
\hline \multirow[t]{2}{*}{ Hermana } & Pre & $120 / 80$ & 37,6 & 0,22 & 0,35 & 0,23 & 1,5 \\
\hline & Post & $96 / 61$ & 17,3 & 2,92 & 0,18 & 0,16 & 1,1 \\
\hline
\end{tabular}

$\mathrm{P}$ Art =presión arterial. AP/ARP =relación aldosterona plasmática/reactividad de renina plasmática. PCRus =proteína $\mathrm{C}$ reactiva ultrasensible. MMP-9 =metaloproteinasa 9. XO =xantina oxidasa. MDA =malondialdehído. VR =valor de referencia. Nota: los resultados en relación al estudio de estrés oxidativo, evaluado mediante xantina oxidasa y malondialdehído se informaron previamente en referencia 21.

normalizaron tanto los niveles de PCRus como los de MMP-9 en todos los sujetos estudiados (Tabla 1).

\section{DISCUSIÓN}

En este estudio reportamos la primera familia portadora de HAF-I debido a una mutación de novo del gen quimérico CYP11B1/CYP11B2. Además encontramos un aumento de los marcadores de inflamación endotelial subclínica, los cuales revirtieron con la normalización de la aldosterona plasmática.

Dado que el HAF-I tiene una herencia autosómica dominante, esperábamos pesquisar en uno de los abuelos paternos del caso índice la presencia del gen quimérico CYP11B1/CYP11B2. En la primera reacción de amplificación del gen quimérico se amplificó un segmento de 3,6 kb, mientras que en la segunda reacción se amplificó un segmento mayor de $4 \mathrm{~kb}$, ubicándose los primers en zonas distintas del gen en cada reacción. De esta manera se descartó la presencia de un polimorfismo en el sitio de unión de primer en la primera reacción, hecho que podría haber sido el causal de no lograr una amplificación adecuada en la abuela o abuelo paternos. Si éste hubiese sido el caso, en la segunda reacción se habría logrado amplificar al sujeto portador de la mutación.
Luego de descartar en los abuelos paternos del caso índice la presencia de polimorfismos en el sitio de unión de primers, se realizó estudio de paternidad entre abuelos y padre del caso índice, encontrándose una correlación perfecta entre las secuencias hipervariables analizadas, certificando la relación parental entre ellos. Por lo tanto, corresponde a la primera familia comunicada a nivel mundial en que se logra demostrar la ocurrencia de una mutación de novo en un miembro de una familia afectada por HAF-I.

En este estudio también se demostró un aumento en los marcadores de inflamación endotelial subclínica en los 3 sujetos estudiados, lo que es concordante con los resultados obtenidos previamente en relación a estrés oxidativo (Tabla $1)^{21}$. Este hallazgo apoya la hipótesis de que la aldosterona estimula directamente la producción a nivel del endotelio de ROS con el consiguiente aumento de la actividad de xantino oxidasa ${ }^{11}$, produciéndose la oxidación de lípidos de membrana lo que lleva a un aumento del MDA ${ }^{12,21}$. Este aumento en el estrés oxidativo llevaría posteriormente a un aumento en la inflamación endotelial subclínica. En relación a estos marcadores, observamos un aumento de MMP-9 en todos los pacientes, mientras que los niveles de PCRus sólo se encontraron elevados en el padre. Estas evidencias llevan a plantear que la inflamación endotelial 
sería un proceso que se produciría después de la producción de ROS y del estrés oxidativo endotelial, requiriendo probablemente un mayor tiempo de exposición a la noxa generada por aldosterona para su desarrollo.

Al ser tratados los pacientes con glucocorticoides, se observó que ambos pacientes tratados con dexametasona normalizaron tanto la AP, ARP y la relación entre ambas. En el caso índice se normalizó la AP, manteniendo una relación AP/ARP levemente elevada. Este hecho se debe probablemente a que en este paciente, por encontrarse aún en etapa de crecimiento, se trató con cortisol, el cual tiene una menor vida media y menor efecto supresor sobre el eje hipotálamo-hipófisis-suprarrenal, no siendo capaz de mantener la ARP en valores normales durante todo el día. Se lograron valores de AP normales en los 3 sujetos, previniendo la exposición del endotelio a niveles suprafisiológicos de aldosterona. Tras el tratamiento se observó una disminución y normalización de los marcadores de inflamación endotelial subclínica, aportando nueva evidencia sobre el efecto deletéreo de la aldosterona sobre el endotelio, el cual sería reversible al normalizarla.

En la hermana del caso índice, si bien se observó un leve aumento en los niveles de PCRus,

\section{REFERENCIAS}

1. Mosso L, Carvajal C, González A, Barraza A, Avila F, Montero J et al. Primary aldosteronism and hypertensive disease. Hypertension 2003; 42: 161-5.

2. Mimez P, Girerd X, Plouin PF, Biacher J, Safar ME, MOURAD JJ. Evidence for an increased rate of cardiovascular events in patients with primary hyperaldosteronism. J Am Coll Cardiol 2005; 45: 1243-8.

3. RidKer P, Hennekens CH, Buring J, Rifai N. C-reactive protein and other markers of inflammation in the prediction of cardiovascular disease in women. $\mathrm{N}$ Engl J Med 2000; 342: 836-43.

4. Sabatine M, Morrow D, Jablonski K, Murguia M, Wayne J, Domanski M et al. Prognostic Significance of the Centers for Disease Control/American Heart Association High-Sensitivity C-Reactive Protein Cut Points for Cardiovascular and Other Outcomes in Patients With Stable Coronary Artery Disease. Circulation 2007; 115: 1528-36.

5. Pitsavos C, Tampourlou M, Panagiotakos DB, Skoumas Y, Chrysohoou C, Nomikos T et aL. Association Between Low-Grade Systemic Inflammation and éstos se mantuvieron dentro del rango normal, presentando además una normalización en los niveles de MMP-9 lo que sugiere una disminución del proceso inflamatorio en ella.

Los glucocorticoides ejercen su efecto antiinflamatorio actuando sobre la expresión de reguladores génicos como son el NFkB y el activador de proteína (AP)-1 14 . Ambos participan en la respuesta inflamatoria en una etapa posterior a la formación de ROS y al aumento de la actividad de xantino oxidasa, por lo que la reducción de marcadores de estrés oxidativo en estos pacientes no estaría influida por el uso de glucocorticoides. La reducción de los marcadores de inflamación endotelial está en relación a la disminución del estrés oxidativo y no al uso de glucocorticoides, si bien no puede ser descartado en forma absoluta algún papel de los glucocorticoides en la disminución de los marcadores inflamatorios.

En conclusión, la elevación de los marcadores de inflamación endotelial subclínica y su posterior normalización con el tratamiento del HAF-I aporta nueva evidencia sobre el posible efecto deletéreo de la aldosterona sobre el endotelio y riesgo cardiovascular. Esta es la primera familia a nivel mundial en la cual se logra demostrar el desarrollo de HAF-I a partir de una mutación de novo.

Type 2 Diabetes Mellitus Among Men and Women from the ATTICA Study. Rev Diabet Stud 2007; 4: 98104.

6. Anan $\mathrm{F}$, Masaki T, Umeno $\mathrm{Y}$, InaO T, Yonemochi $\mathrm{H}$, Eshima N ET AL. Correlations of high-sensitivity Creactive protein and atherosclerosis in Japanese type 2 diabetic patients. Eur J Endocrinol 2007; 157: 311-7.

7. Bittner A, Castro P, Pérez O, Corbalán R, Troncoso R, Chiong M et AL. Inflamación y estrés oxidativo en el síndrome coronario agudo: ¿dos fenómenos relacionados? Boletín de la Escuela de Medicina Universidad Católica de Chile 2005; 30: 6-11.

8. Schönbeck U, Mach F, Sukhova G, Murphy C, Bonnefoy J, FABUNMI R ET AL. Regulation of matrix metalloproteinase expression in human vascular smooth muscle cells by T lymphocytes. A role for CD40 signaling in plaque rupture? Circ Res 1997; 81: 448-54.

9. PACKARD R, LibBy P. Inflammation in Atherosclerosis: From Vascular Biology to Biomarker Discovery and Risk Prediction. Clin Chem 2008; 54: 24-38.

10. Blankenberg S, Rupprecht H, Poirier O, Bickel C, Smieja, HafNer G et al. Plasma concentrations and 
genetic variation of matrix metalloproteinase 9 and prognosis of patients with cardiovascular disease. Circulation 2003; 107: 1579-85.

11. Landmesser U, Drexler H. Endothelial function and hyertension. Curr Opin Cardiol 2007; 22: 316-20.

12. Ozkul A, Akyol A, Yenisey C, Arpaci E, Kiylioglu N, Tataroglu C. Oxidative stress in acute ischemic stroke. J Clin Neurosci 2007; 14: 1062-6.

13. Glowinska-OlszewsKa B, Urban M. Elevated matrix metalloproteinase 9 and tissue inhibitors of metalloproteinase 1 in obese children and adolescents. Metab Clin Exp 2007; 56: 799-805.

14. Rahman I, AdCOCK IM. Oxidative stress and redox regulation of lung inflammation in COPD. Eur Resp J 2006; 28: 219-42.

15. Chai W, Danser J. Why are mineralocorticoid receptor antagonists cardioprotective? NaunynSchmiedeberg's Arch Pharmacol 2006; 374: 153-62.

16. AHokAs RA, Sun Y, Bhattacharya SK, GerLing IC, WEBER KT. Aldosteronism and a proinflammatory vascular phenotype: role of $\mathrm{Mg}^{2+}, \mathrm{Ca}^{2+}$, and $\mathrm{H}_{2} \mathrm{O}_{2}$ in peripheral blood mononuclear cells. Circulation 2005; 111: 51-7.

17. Yoshimoto T, Hirata Y. Aldosterone as a cardiovascular risk hormone. Endocr J 2007; 54: 359-70.

18. Jackson RV, Lafferty A, Torpy DJ, Stratakis C. New genetic insights in familial hyperaldosteronism. Ann NY Acad Sci 2002; 970: 77-88.
19. Lfton RP, Dumy RG, Powers M, Rich GM, Cook S, Uuck S ET AL A chimenic 11b-hydroxylase/aldosterone synthase gene causes glucocorticoid-remediable aldosteronism and human hypertension. Nature 1992; 355: 262-5.

20. Pascoe L, Curnow K, Slutsker L, Connell JM, Speiser PW, NeW MI ET AL. Glucocorticoid-suppressible hyperaldosteronism results from hybrid genes created by unequal crossover between CYP11B1 and CYP11B2. Proc Natl Acad Sci USA 1992; 89: 8327-31.

21. Stehr C, Carvajal C, Alcaíno H, Lacourt P, Cattani A, Mosso L ET AL. Variabilidad fenotípica y estrés oxidativo endotelial en una familia con hiperaldosteronismo familiar tipo I. Rev Chil Endocrinol Diabetes 2008; 1: 18-23.

22. Kameda K, Matsunaga T, Abe N, Hanada $H$, Ishizaka $H$, ONO H ET AL. Correlation of oxidative stress with activity of matrix metalloproteinase in patients with coronary artery disease. Possible role for left ventricular remodelling. Eur Heart J 2003; 24: 2180-5.

23. Jonsson J, Kiemm S, Tunny T, Stowasser M, Gordon R. A new genetic test for familial hyperaldosteronism type I aids in the detection of curable hypertension. Biochem Bioph Res Comm 1995; 207: 565-71.

24. Macconnachie A, Kelly K, Mcnamara A, Loughun S, Gates L J, Ingus GC ET AL. Rapid diagnosis and identification of cross-over sites in patients with glucocorticoid remediable aldosteronism. J Clin Endocrinol Metab 1998; 83: 4328-31. 